\title{
Tratamiento de malformaciones y tumores vasculares, en un centro de referencia en Bogotá
}

\author{
Treatment of vascular malformations and tumors in a reference \\ center in Bogotá
}

\author{
Cesar E. Jiménez ${ }^{1}$ D, Leonardo Randial² $\mathbb{D}$, Iván Silva², Manuel Hossman², Juan David Rueda ${ }^{3}$, \\ Fernando Quiroga ${ }^{4} \mathbb{D}$
}

1 Médico, especialista en Cirugía vascular; jefe, servicio de Cirugía Vascular Periférica, Hospital Universitario Clínica San Rafael, Bogotá, D.C., Colombia.

Médico, especialista en Cirugía vascular, Hospital Universitario Clínica San Rafael, Bogotá, D.C., Colombia.

Médico, servicio de Cirugía Vascular Periférica, Hospital Universitario Clínica San Rafael, Bogotá, D.C., Colombia.

4 Médico, especialista en Epidemiología, Fundación Sinergia, Hospital Universitario Clínica San Rafael, Bogotá, D.C., Colombia.

\section{Resumen}

Introducción. Las malformaciones vasculares son anomalías que están presentes desde el nacimiento, no desaparecen y pueden crecer a lo largo de la vida. Se ha demostrado en estudios retrospectivos que la terminología para clasificar las anomalías vasculares es inexacta en un $69 \%$ de casos, por lo que se hace un diagnostico inadecuado y en un $53 \%$ de casos se brinda al paciente y su familia una información incorrecta del tratamiento y el curso clínico.

Métodos. Estudio prospectivo longitudinal, realizado entre 2016 y 2019, donde se incluyeron pacientes con anomalías vasculares, que consultaron a nuestra institución, fueron valorados por el servicio de cirugía vascular, se hizo un plan diagnóstico y manejo integral, vía endovascular, quirúrgica o mixta, de acuerdo con cada caso.

Resultados. La malformación más común fue la de tipo venoso, en el 40,3\% de los casos. Se realizó manejo endovascular en el 93,I \% de casos de malformaciones vasculares y quirúrgico en el 6,9 \%. La mejoría de los síntomas que motivaron la consulta fue del $100 \%$ para los tumores vasculares y del 70,8 \% para las malformaciones. Sin embargo, los resultados son heterogéneos.

Discusión. Es necesario realizar un adecuado diagnóstico de las anomalías vasculares, para alcanzar un tratamiento eficaz, con mejoría de los síntomas.

Palabras clave: malformaciones vasculares; neoplasias de tejido vascular; procedimientos quirúrgicos vasculares; prótesis vascular; injerto vascular; procedimientos endovasculares; embolia.

Fecha de recibido: 02/10/2019 - Fecha de aceptación: 10/02/2020

Correspondencia: Cesar Eduardo Jiménez, Calle 17 \# 16-68, casa 18, Chía, Colombia, Teléfono: 3142976816

Correo electrónico: cesarejmd@yahoo.com

Citar como: Jiménez CE, Randial L, Silva I, Hossman M, Rueda JD, Quiroga F. Tratamiento de malformaciones y tumores vasculares, en un centro de referencia en Bogotá. Rev Colomb Cir. 2020;35:647-58. https://doi.org/10.30944/20117582.803

Este es un artículo de acceso abierto bajo una Licencia Creative Commons - BY-NC-ND https://creativecommons.org/licenses/by-ncnd/4.0/deed.es 


\begin{abstract}
Introduction. Vascular malformations are abnormalities that are present from birth, do not disappear and can grow throughout life. It has been shown in retrospective studies that the terminology to classify vascular anomalies is inaccurate in $69 \%$ of cases, an inappropriate diagnosis is made and in $53 \%$ of cases the patient and their family are given incorrect information on the treatment and the clinical course.
\end{abstract}

Methods. Longitudinal prospective study conducted between 2016 and 2019. Patients with vascular anomalies and consulted to our institution were included. They were evaluated by the vascular surgery service, and a comprehensive diagnosis and management plan was made, including endovascular, surgical or mixed, according to each case.

Results. The most common malformation was the venous type in $40.3 \%$ of the cases. Endovascular management was performed in $93.1 \%$ of cases and surgery in $6.9 \%$. The improvement in the symptoms that led to the consultation was $100 \%$ for vascular tumors and $70.8 \%$ for malformations. However, the results are heterogeneous.

Conclusions. It is necessary to obtain an adequate diagnosis of vascular anomalies, to achieve an effective treatment, with improvement of the symptoms.

Keywords: vascular malformations; neoplasms, vascular tissue; vascular surgical procedures; blood vessel prosthesis; vascular grafting; endovascular procedures; embolism.

\section{Introducción}

Las malformaciones vasculares son anomalías que están presentes desde el nacimiento y, contrario a los hemangiomas, no desaparecen; incluso, pueden crecer a lo largo de la vida del ser

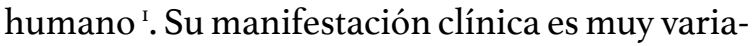
ble, desde una mancha asintomática que afecta únicamente desde el punto de vista estético, hasta lesiones de alto flujo o localizaciones particulares, que pueden poner en peligro la vida de quien la padece ${ }^{1,2}$. El tratamiento de estas enfermedades relativamente raras es difícil, más aún cuando no hay una suficiente experiencia que permita orientar de forma adecuada el diagnóstico, la clasificación y el tratamiento de la patología. Es imprescindible contar con un tratamiento con enfoque multidisciplinario y seguimiento de los pacientes con anomalías vasculares ${ }^{1,2}$.

La ISSVA (International Society for the Study of Vascular Anomalies) es la agremiación internacional para el estudio y tratamiento de estas enfermedades, fundada en 1992 y clasifica las anomalías vasculares en tres grandes grupos: $i$. Malformaciones vasculares. ii. Tumores vasculares y iii. Complejos sindromáticos ${ }^{3-5}$.
Las malformaciones vasculares han generado confusión en diferentes áreas de la medicina, debido a su baja prevalencia, el escaso conocimiento por parte de los profesionales de la salud, las múltiples especialidades que atienden a los pacientes, y la nomenclatura confusa y en muchas ocasiones equivocada para catalogarlas. Esto lleva a que estas entidades sean mal diagnosticadas o no reciban el manejo adecuado.

Se ha demostrado en estudios retrospectivos que la terminología usada para catalogar las anomalías vasculares es incorrecta en el $69 \%$ de los casos, dándole al paciente un diagnostico inadecuado de su enfermedad, y en el $53 \%$ de los casos se le da a la familia y al paciente una información inadecuada en relación con el tratamiento y la evolución de su anomalía vascular ${ }^{3}$. En otros estudios, el término hemangioma se ha usado de manera inequívoca en 7I,3\% de artículos médicos, independientemente de la especialidad que se trate, y al menos el $20 \%$ de los pacientes recibieron un tratamiento incorrecto para su enfermedad, por un diagnostico impreciso ${ }^{4,5}$.

Este estudio tuvo como propósito determinar la eficacia del tratamiento de anomalías vascula- 
res en nuestra institución, una entidad de cuarto nivel de complejidad de referencia del Distrito Capital de Bogotá, a partir de un adecuado diagnóstico y clasificación de las mismas.

\section{Métodos}

Estudio prospectivo, longitudinal y descriptivo de una sola cohorte, realizado entre los años 2016 y 2019, que incluyó pacientes con anomalías vasculares que consultaron a nuestro servicio de Cirugía Vascular Periférica del Hospital Universitario Clínica San Rafael, Bogotá, D.C., Colombia, y se decidió un plan diagnóstico y de manejo de acuerdo con cada caso.

Se analizaron las siguientes variables: edad, sexo, diagnóstico inicial de remisión, tipo de malformación, motivo de consulta, tipo de procedimiento realizado, numero de procedimientos realizados a cada paciente, material usado, complicaciones intra y postoperatorias, mejoría de los síntomas, desaparición o disminución de la anomalía vascular 3 meses después de la intervención y mortalidad a 30 días.

Se clasificaron las anomalías vasculares de acuerdo con la ISSVA ${ }^{4,5}$. En todos los casos se realizó el examen clínico del paciente y para la evaluación imagenológica los más utilizados fueron el dúplex vascular y la resonancia nuclear magnética. Los procedimientos invasivos como arteriografía y flebografía se usaron como métodos diagnósticos y terapéuticos al mismo tiempo.

Las indicaciones de tratamiento estuvieron orientadas por los síntomas que presentaban los pacientes. Los procedimientos se realizaron por uno o dos cirujanos vasculares, en sala de hemodinamia, bajo visión fluoroscópica y con ayuda de ultrasonido vascular dú plex color siempre. Se utilizó anestesia general para los casos que se usó alcohol u Onyx ${ }^{\circledR}$; para los casos de escleroterapia con otros agentes se utilizó anestesia local únicamente. Se accedió por vía de arteria femoral para los casos de malformaciones arteriovenosas y por vía directa percutánea para las venosas; en algunos casos por ambas vías. Se siguió clínicamente, y en algunos casos con imagenología, a todos los pacientes durante un periodo de 3 meses después de realizado el procedimiento.

Se estimaron valores absolutos y relativos para la descripción de las variables y se empleó el estadístico $\mathrm{chi}^{2}$ para el análisis bivariado de diferencia de proporciones.

\section{Resultado}

Entre los años 2016 y 2019, se presentaron 88 pacientes con anomalías vasculares, 72 pacientes con malformaciones vasculares, 7 con tumores vasculares y 9 con complejos sindromáticos (tabla I). El diagnóstico inicial de remisión, con el cual llegó rotulado el paciente a nuestra valoración, fue de hemangioma en el $68 \%$ de los casos, malformación venosa en el 6,9\%, tumor vascular, malformación linfática y fistula arteriovenosa en el 4,2 \% cada una. El motivo de consulta para las malformaciones vasculares fue dolor en el 38,9\% de los casos, masa no pulsátil en 30,6 \%, masa pulsátil en $13,9 \%$, sangrado en 9,7 \% y deformación estética en 6,9\%; mientras para los tumores vasculares el motivo fue masa no pulsátil en el 7I,4 \% de casos y dolor en el 28,6\%.

Las malformaciones vasculares tuvieron igual distribución por sexo, mientras los tumores vasculares se presentaron más en hombres (57\%). La edad promedio de presentación para las malformaciones vasculares fue de 29 años (rango: I-65 años) y para los tumores fue de 34 años (rango: 5-65 años).

El tipo de malformación vascular más común fue la venosa en el 40,3\% de los casos, seguida de la fistula arteriovenosa (33,3\%), la linfática (I2,5\%) y los complejos sindromáticos (II,I \%), siendo el de Klippel-Trenaunay el más común. Las malformaciones se localizaron en los miembros inferiores en un $5 \mathrm{I}, 4 \%$, miembros superiores, I8,I \%; abdomen, I5,3 \%; cara, 6,9 \%; cuello, 4,2 \%, y tórax, 4,2 \%. Los tumores vasculares se ubicaron en miembros inferiores en el $57 \%$; miembros superiores, $28 \%$, y abdomen $14 \%$.

Se practicaron en total IO2 procedimientos. Para el manejo de las malformaciones vasculares se realizó un procedimiento en el 58,3\% de los casos y dos en el $27,8 \%$, y de manera similar, para los tumores vasculares se realizó un pro- 
Tabla 1. Aspectos demográficos y caracterización de las anomalías.

\begin{tabular}{|c|c|c|c|c|}
\hline & & $\begin{array}{l}\text { Malformación } \\
\text { n (\%) }\end{array}$ & $\begin{array}{l}\text { Tumor } \\
\mathbf{n}(\%)\end{array}$ & $\begin{array}{c}\text { Sindromáticos } \\
\mathrm{n}(\%)\end{array}$ \\
\hline \multicolumn{2}{|c|}{ Anomalías vasculares } & 72 & 7 & 9 \\
\hline \multirow[t]{3}{*}{ Edad en años } & Mínimo & 1 & 5 & 23 \\
\hline & Máximo & 65 & 65 & 61 \\
\hline & Promedio & 29.6 & 34.3 & 39.5 \\
\hline \multirow[t]{2}{*}{ Sexo } & Hombre & $36(50 \%)$ & $3(42,9 \%)$ & $4(44.4 \%)$ \\
\hline & Mujer & $36(50 \%)$ & $4(57,1 \%)$ & $5(55.6 \%)$ \\
\hline \multirow{6}{*}{$\begin{array}{l}\text { Tipo de } \\
\text { malformación }\end{array}$} & Venosa & $29(40,3 \%)$ & & \\
\hline & Fistula arterio-venosa & $24(33,3 \%)$ & & \\
\hline & Linfática & $9(12,5 \%)$ & & \\
\hline & Sindromática & $8(11,1 \%)$ & & $9(100 \%)$ \\
\hline & Linfática o veno- linfática & $1(1,4 \%)$ & & \\
\hline & $\begin{array}{l}\text { Fistula arterio-venosa y } \\
\text { venoembrionarias }\end{array}$ & $1(1,4 \%)$ & & \\
\hline \multirow{5}{*}{$\begin{array}{l}\text { Motivo } \\
\text { de consulta }\end{array}$} & Dolor & $28(38,9 \%)$ & $2(28,6 \%)$ & $2(22.2 \%)$ \\
\hline & Masa no pulsátil & $22(30,6 \%)$ & $5(71,4 \%)$ & \\
\hline & Masa pulsátil & $10(13,9 \%)$ & 0 & \\
\hline & Sangrado & $7(9,7 \%)$ & 0 & \\
\hline & Deformidad estética & $5(6,9 \%)$ & 0 & $7(77.8 \%)$ \\
\hline \multirow{5}{*}{$\begin{array}{l}\text { Diagnóstico } \\
\text { ilnicial }\end{array}$} & Hemangioma & $49(68,1 \%)$ & $6(85,7 \%)$ & \\
\hline & Enfermedad venosa & $5(6,9 \%)$ & $1(14,3 \%)$ & $5(55.6 \%)$ \\
\hline & Fistula arterio-venosa & $3(4,2 \%)$ & 0 & $2(21.2 \%)$ \\
\hline & Enfermedad linfática & $3(4,2 \%)$ & 0 & $2(21.2 \%)$ \\
\hline & Tumor vascular & $3(4,2 \%)$ & 0 & \\
\hline
\end{tabular}

cedimiento en el 57,I \% de los casos y dos en el $28,6 \%$ (tabla 2).

Se presentaron complicaciones intraoperatorias en tres pacientes con malformaciones $(4,2 \%)$ sometidos a manejo endovascular, dos por sangrado y uno por hipertensión pulmonar aguda; no hubo complicaciones intraoperatorias en los pacientes con tumores vasculares.

Se realizó seguimiento clínico por tres meses después de la intervención, y se encontró una complicación posterior al procedimiento (sangrado) en un paciente con malformación; en pacientes con tumores no se registraron complicaciones postoperatorias. Se logró la oclusión de los vasos malformados por técnicas endovasculares (considerado "éxito técnico") para el tratamiento de malformaciones en el 98,6\% y resección quirúrgica completa del tumor en el IOO\% de casos. El uso de alcohol se asocia a cambios dérmicos por necrosis o alteraciones de la coloración, por eso estas no se consideran complicaciones, ya que son parte del efecto de este agente; se presentaron en algunos de los pacientes, sin embargo, se resolvieron espontáneamente sin necesidad de otra intervención adicional.

Con respecto a la mejoría de los síntomas de motivo de consulta, se encontró que para los tumores vasculares en el Ioo \% de los casos se resolvieron y para las malformaciones, en el 70,8 \%. La disminución de la alteración observada por el paciente o su familia fue del $65,3 \%$ en malformaciones vasculares y del Ioo \% en tumores. No se presentó mortalidad a 30 días en la cohorte estudiada (tabla 3).

Se encontró una diferencia estadísticamente significativa $(\mathrm{p}<0.000)$ en la mejoría de la sintomatología respecto del motivo de consulta, el dolor, la masa pulsátil, la masa no pulsátil y el sangrado. Aunque la deformidad estética mejoró para 3 de los 5 pacientes, la diferencia no fue estadísticamente significativa (tabla 4). 
Tabla 2. Descripción de los procedimientos practicados y sus complicaciones

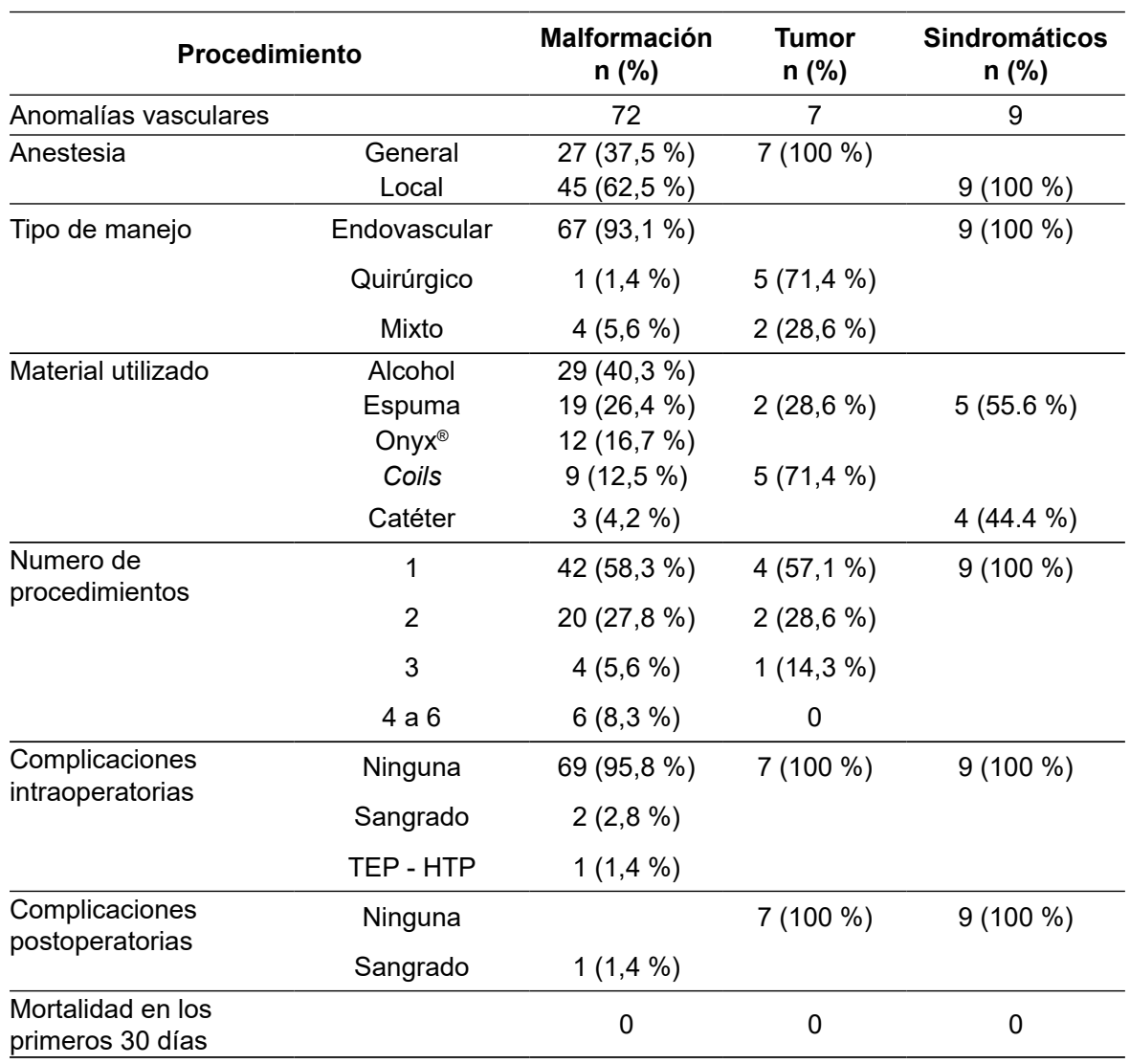

TEP: tromboembolismo pulmonar; HTP: hipertensión pulmonar

Tabla 3. Mejoría de la sintomatología con el manejo realizado según el motivo de consulta

\begin{tabular}{lcccc}
\hline \multirow{2}{*}{ Motivo de consulta } & \multicolumn{4}{c}{ Mejoría del síntoma motivo de consulta } \\
\cline { 2 - 5 } & \multicolumn{3}{c}{ NO } & \multicolumn{2}{c}{ SI } \\
\cline { 2 - 5 } & $\mathbf{n}$ & \% del $\mathbf{n}$ de fila & $\mathbf{n}$ & $\%$ del $\mathbf{n}$ de fila \\
\hline Deformidad estética & 2 & $40 \%$ & 3 & $60 \%$ \\
Dolor & 9 & $30 \%$ & 21 & $70 \%$ \\
Masa no pulsátil & 7 & $25,9 \%$ & 20 & $74,1 \%$ \\
Masa pulsátil & 2 & $20 \%$ & 8 & $80 \%$ \\
Sangrado & 1 & $14,3 \%$ & 6 & $85,7 \%$ \\
\hline
\end{tabular}

Tabla 4. Seguimiento durante los primeros tres meses después de la intervención

\begin{tabular}{lccc}
\hline & $\begin{array}{c}\text { Malformación } \\
\mathbf{n ~ ( \% )}\end{array}$ & $\begin{array}{c}\text { Tumor } \\
\mathbf{n ~ ( \% )}\end{array}$ & $\begin{array}{c}\text { Sindromáticos } \\
\mathbf{n}(\mathbf{\%})\end{array}$ \\
\hline Anomalías vasculares & 72 & 7 & 9 \\
Éxito técnico & $71(98,6 \%)$ & $7(100 \%)$ & $9(100 \%)$ \\
Mejoría de los síntomas respecto & $51(70,8 \%)$ & $7(100 \%)$ & $7(77.8 \%)$ \\
al motivo de consulta & $47(65,3 \%)$ & $7(100 \%)$ & $2(22.2 \%)$ \\
Disminución de la malformación & $8(11,1 \%)$ & $3(42,9 \%)$ & 0 \\
Desaparición de la malformación & & & \\
\hline
\end{tabular}




\section{Discusión}

En el año I988 tiene lugar la denominada clasificación de Hamburgo (posteriormente homologada por la ISSVA en 1992), donde se dividieron las anomalías vasculares en malformaciones vasculares, tumores vasculares y complejos sindromáticos ${ }^{4-7}$. Existe confusión en el diagnóstico y la clasificación de las anomalías vasculares, por lo que es frecuente que se cataloguen los pacientes de manera errónea. En este trabajo se encontró que el $68 \%$ de casos venían diagnosticados de manera incorrecta, ya fuera por clínica o por imágenes, como hemangioma.

Fueron tratados pacientes de diferentes edades, desde infantes de meses de vida hasta adultos mayores de 65 años, sin encontrar preponderancia según el sexo. La anomalía vascular más frecuente encontrada en nuestro estudio fue la malformación de tipo venoso (40 \% de casos), que concuerda con la casuística mundial, donde corresponden a un $70-80 \%$ de los casos ${ }^{8,9}$.

Las malformaciones linfáticas, que la literatura denomina higroma quístico o linfagioma, se diagnosticaron en $\mathrm{I} 2,5 \%$ de casos, la mayoría en niños pequeños, y su localización más frecuente es la cabeza y el cuello (figura I). Tienen mayor recidiva con manejo quirúrgico y mejores resultados con los procedimientos percutáneos ${ }^{\mathrm{IO}}$ (figura 2).

En este estudio las malformaciones arteriovenosas fueron la segunda anomalía vascular
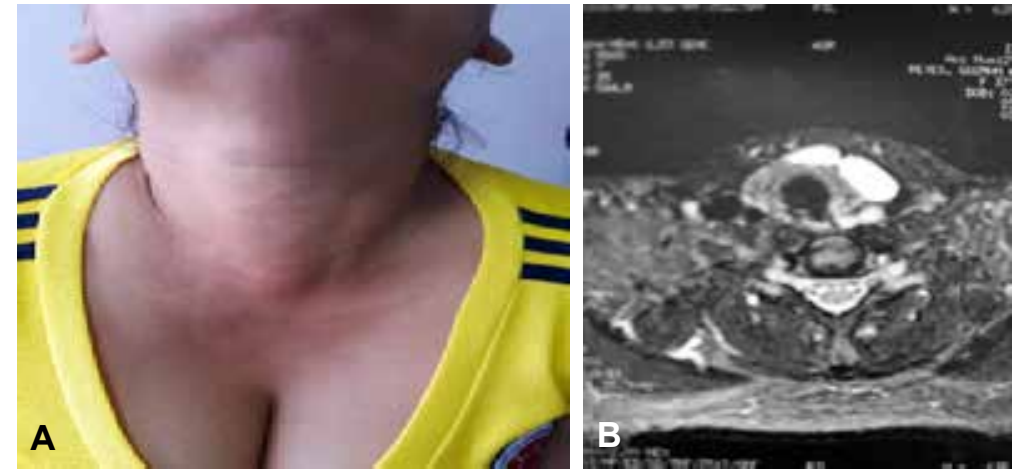

Figura 1. Malformación linfática en cuello. A) Aspecto al examen físico donde se aprecia que la masa aumenta con las maniobras de Valsalva. B) Resonancia nuclear que muestra hipercaptación de lesión linfática.
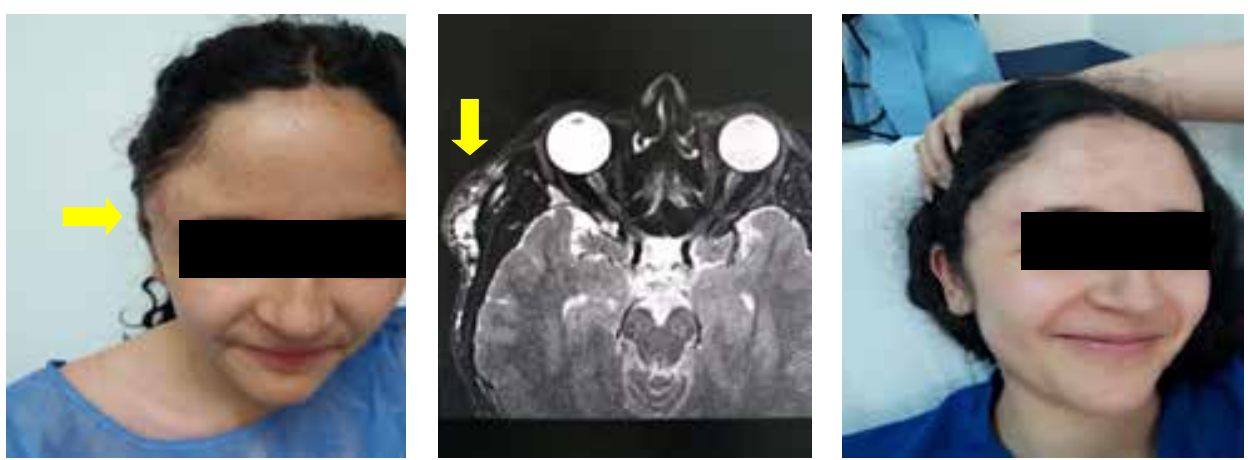

Figura 2. Paciente con malformación vascular en región temporal derecha con deformidad estética y dolor, manejada con alcohol absoluto vía percutánea. A) Aspecto previo a la intervención. B) Resonancia magnética nuclear donde se aprecia la lesión. C) Aspecto de la paciente al segundo mes después del tratamiento. 
en frecuencia de presentación (33,3\%), y se manifestaron en forma de masa pulsátil (figura 3). Estas malformaciones hacen referencia a trayectos fistulosos de alta velocidad y baja resistencia, que comunican de forma anormal arterias con venas ${ }^{11,12}$.

Entre los 9 pacientes con complejos sindromáticos, el síndrome de Klippel-Trenaunay fue el preponderante (II,I \%). Esta entidad se caracteriza por la triada clásica de hemihipertrofia de la extremidad, manchas de vino oporto y anomalías vasculares de predominio venoso, linfático o mixtas; se ve principalmente en los miembros inferiores, pero también se puede presentar en los miembros superiores ${ }^{13-16}$ (figura 4). Nuestros pacientes requirieron manejo quirúrgico y endovascular, resaltando el uso de ablación endovascular por radiofrecuencia de venas anómalas.

Los tumores diagnosticados fueron hemangiopericitoma y hemangioma verrugoso. Son realmente entidades infrecuentes, que pueden surgir desde el nacimiento o aparecer en algún momento de la vida. Los más frecuentes son el hemangioma de los recién nacidos y el hemangioma hepático, pero no tuvimos esta casuística en nuestro estudio ${ }^{17-19}$ (figura 5).

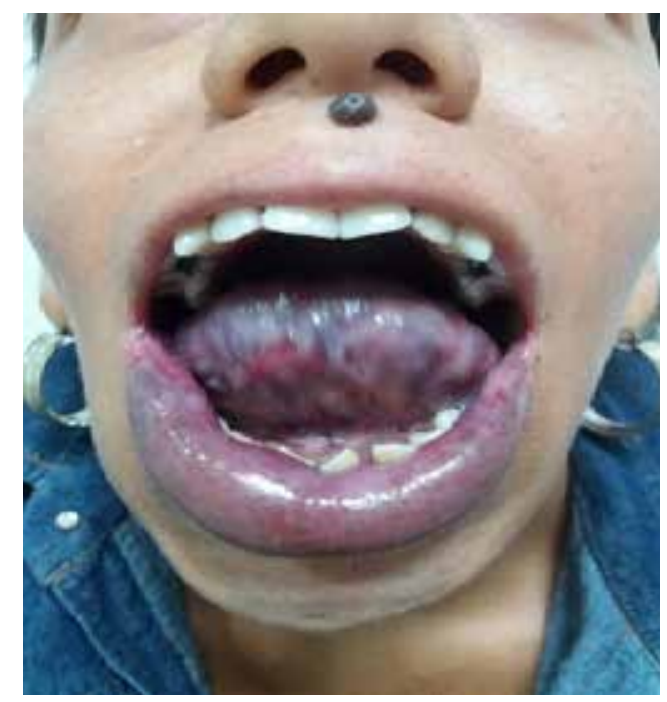

Figura 3. Paciente con fistulas arteriovenosas en boca que generan deformación de la cavidad oral y desplazamiento dental

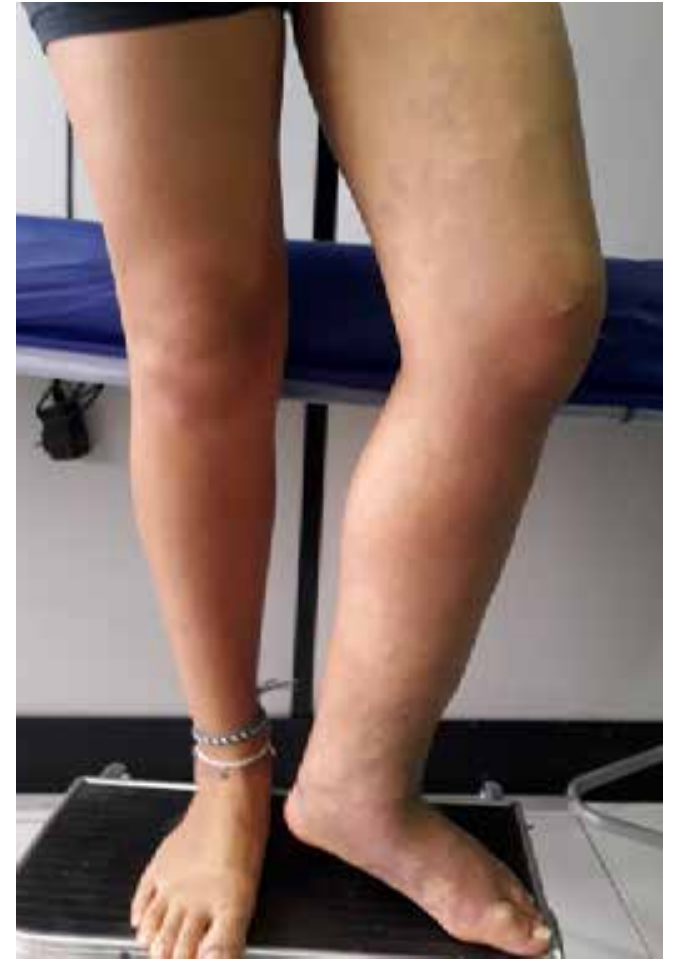

Figura 4. Paciente con síndrome de Klippel-Trenaunay (hemihipertrofia, manchas de vino oporto y malformaciones venolinfáticas) con compromiso de miembro inferior izquierdo.

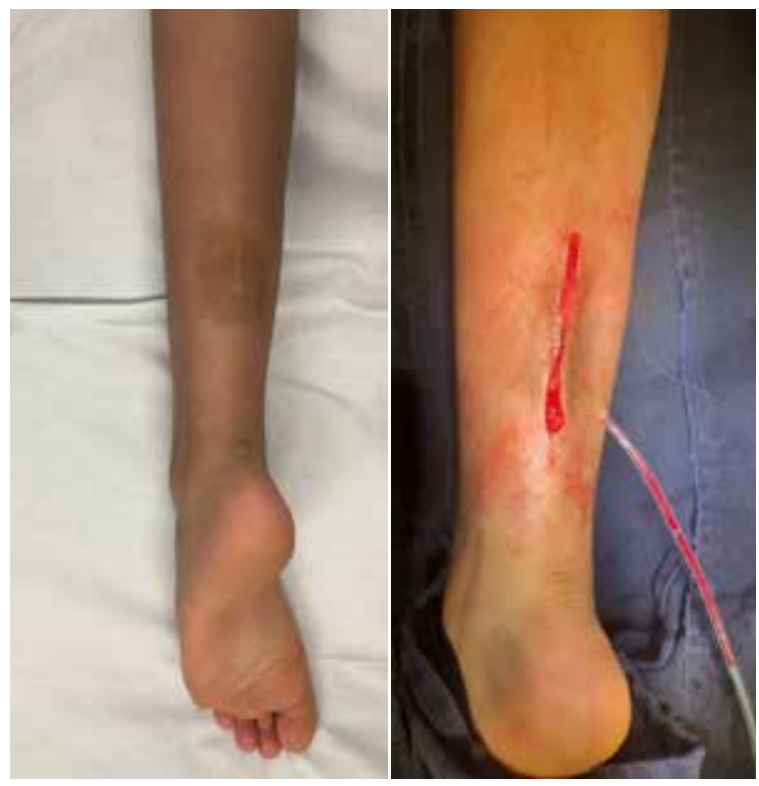

Figura 5. Glomangioma en pierna izquierda, que fue tratado con resección quirúrgica amplia. 
El diagnóstico y el tratamiento de las anomalías vasculares son un reto complejo, debido a la diversidad en las lesiones, que se presentan a diferentes edades, con variados síntomas y opciones de manejo (medico, quirúrgico, endovascular, mixto). La primera evaluación, y la más importante, es la que se hace durante el examen físico, determinando las características de la lesión, localización, tamaño y compromiso de estructuras vecinas o vitales. De acuerdo con los síntomas y la localización, los exámenes indicados van a incluir rayos $\mathrm{X}$ simples, dúplex vascular, o resonancia nuclear magnética. Los estudios invasivos, como arteriografía y flebografía, son de suma importancia ya que sirven para hacer el diagnostico anatómico y, al mismo tiempo, para realizar el tratamiento con técnicas endovasculares ${ }^{20-22}$ (figura 6).

Únicamente se trataron aquellos pacientes sintomáticos, con complicaciones secundarias a su malformación (sangrado, dolor crónico, discapacidad funcional o falla cardiaca), con lesiones que pueden comprometer la vida si crecen dada su localización (ojos, vía aérea, tracto gastrointestinal), o con indicación estética. En este trabajo se encontró que, en los casos de malformaciones vasculares, el dolor y la masa fueron los principales síntomas y el motivo de intervención en más del $60 \%$ de los casos. Entre más temprano se trate la malformación, mejor será el resultado y menores las complicaciones ${ }^{23-25}$.

El hallazgo más significativo de este estudio radicó en que la mayoría de los pacientes mejoraron con el tratamiento, en un porcentaje que se considera alto dado el cuadro clínico que los pacientes tenían antes de consultar. Las anomalías vasculares en muchas ocasiones no se pueden tratar de manera completa con una sola intervención, ya que son muy complejas, por eso es necesario informar al paciente y la familia al respecto. Adicionalmente, se pueden utilizar técnicas mixtas, endovasculares y quirúrgicas, para obtener el mejor resultado ${ }^{26-28}$. En el presente estudio, los tumores vasculares se manejaron por vía quirúrgica en la mayoría de los casos, pero se utilizaron procedimientos endovasculares

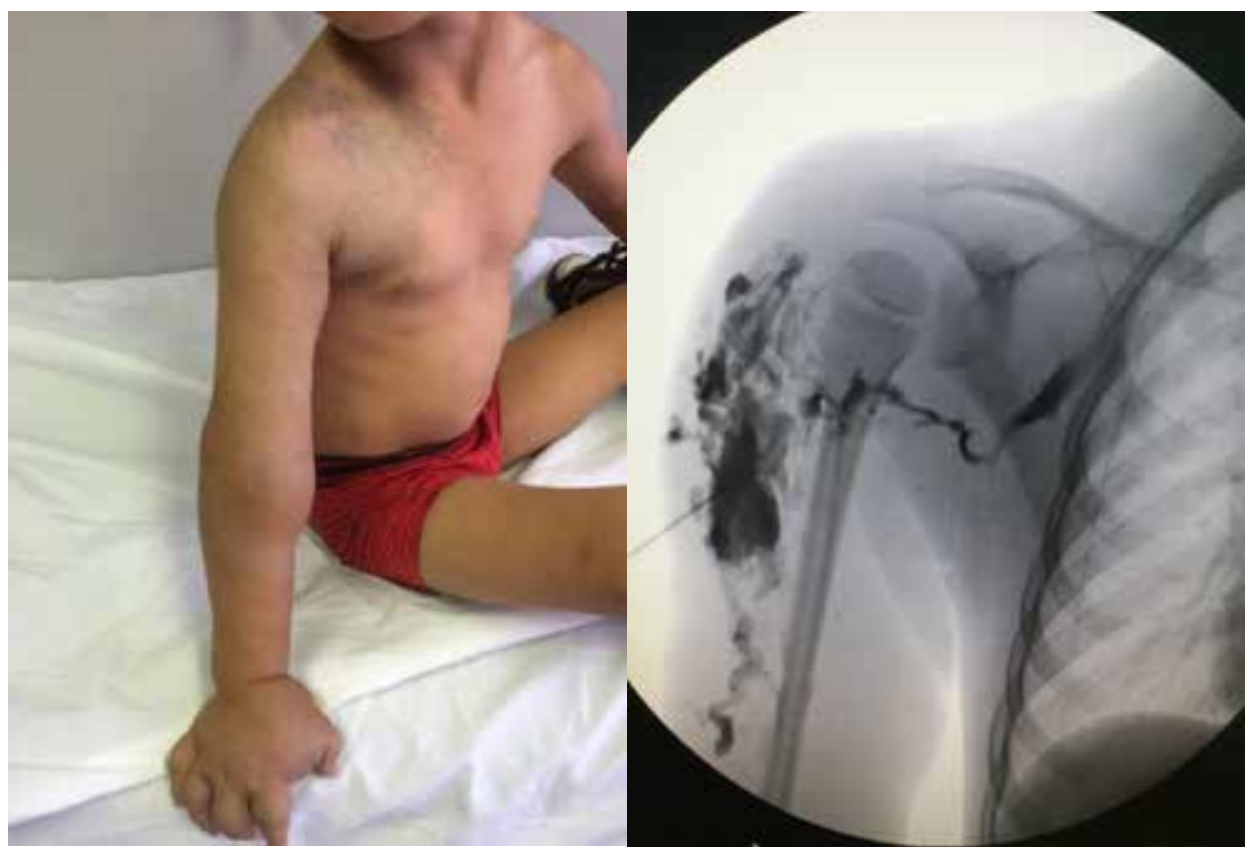

Figura 6. Paciente con lagos venosos malformados que drenan a vena profunda normal, manejado con alcohol y espirales de platino (coils). 
previos para disminuir su tamaño y el sangrado intraoperatorio.

Los procedimientos endovasculares se realizaron en tiempo real con el uso de fluoroscopia dinámica y dúplex vascular. Se deben usar los dos métodos para obtener resultados óptimos y disminuir complicaciones; las embolizaciones guiadas sólo por dúplex no son adecuadas y pueden ser peligrosas, por eso el uso de fluoroscopia es mandatorio ${ }^{29,30}$ (figura 7). Los procedimientos guiados por fluoroscopia se vienen utilizando desde hace más de 20 años y el refinamiento de las técnicas y la tecnología disponible ha hecho posible que las malformaciones se puedan tratar con estos métodos. Puede que la mayoría no sean curables, pero la mejoría clínica y estética son importantes.

Están disponibles diferentes dispositivos y medicamentos. En este estudio, el alcohol absoluto al $99 \%$ se utilizó en el 40,3\% de los pacientes, pues es el mejor agente esclerosante

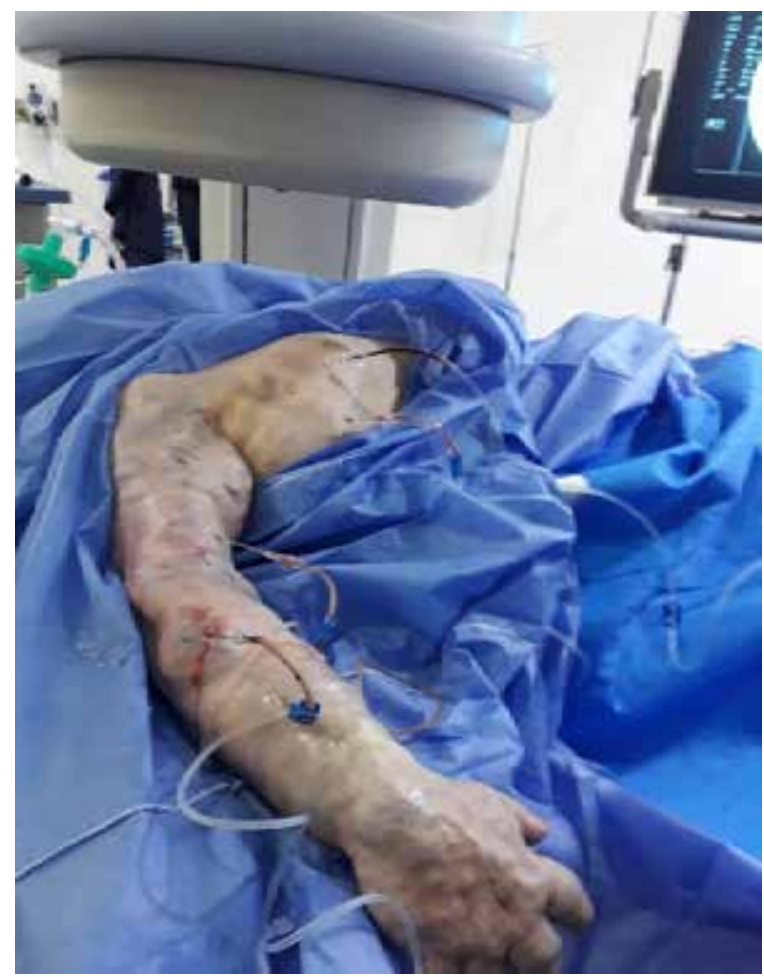

Figura 7. Paciente con múltiples malformaciones en brazo derecho, que recibió punciones para inyección de alcohol. por su potencia y facilidad de uso, pero se debe tener experiencia y cuidado en su utilización ${ }^{31,32}$. Los agentes esclerosantes de baja potencia que se administran en forma de espuma, como el polidocanol o el clorhidrato de lapidium, se usaron solo en el $5 \%$ de los casos, debido a su baja potencia, recidiva y pobres resultados en vasos malformados de gran tamaño 33,34 . Los agentes líquidos para producir émbolos, cuyo principal representante es Onyx ${ }^{\circledR}$, un copolímero de etileno-vinil alcohol disuelto en dimetil sulfóxido, indicado en los casos de fistulas arteriovenosas de mediano y alto flujo, se utilizaron en el I6,7\% de los casos ${ }^{35-37}$.

Los coils, que se colocaron en el I2,5\% de los casos, son alambres de platino o acero inoxidable, útiles como coadyuvantes en la oclusión de vasos nutricios, pero que mal empleados pueden generar empeoramiento de las lesiones ${ }^{38,39}$. Las microesferas y micropartículas no se emplearon en ningún caso, pero están descritas en el tratamiento de algunas anomalías vasculares, aunque por su rápida aglutinación y fácil circulación sistémica pueden tener complicaciones; si se utilizan, deben acompañarse de otros métodos de oclusión endovascular ${ }^{40,41}$. Algunos medicamentos, como la bleomicina o la tetraciclina, se han usado especialmente para el manejo de malformaciones linfáticas, pero su índice de complicaciones no es despreciable, por lo que se recomienda el uso de otras alternativas ${ }^{42-44}$.

Finalmente, es de anotar que no se presentó mortalidad a 30 días y la incidencia de complicaciones fue del $5 \%$. El $70 \%$ de los pacientes mejoraron de sus síntomas. Consideramos que estos buenos resultados son producto de la experiencia que se adquiere en la medida que se cuenta con mayor caustica y se ejecutan los protocolos aplicados de manera sistemática.

\section{Conclusión}

Un adecuado diagnóstico de las malformaciones y los tumores vasculares definen de forma importante el éxito del tratamiento. Este estudio permitió mostrar que el $68 \%$ de los pacientes incluidos llegaron a este centro de atención con un 
diagnóstico incorrecto y con planes de manejo inadecuados, lo que contribuye a menoscabar la calidad de vida de los pacientes y sus familias.

Por su parte, este trabajo demuestra la eficacia del tratamiento de las anomalías vasculares en nuestro servicio, con mejoría de síntomas, desaparición de las anomalías y mínima morbimortalidad.

Dada la heterogeneidad de las anomalías y los tratamientos, se requieren más estudios que incluyan un mayor número de pacientes, con el fin de obtener más información sobre estas entidades, para poder socializar con la comunidad médica sobre la importancia del adecuado diagnóstico en estos pacientes, para alcanzar el éxito en el tratamiento de las anomalías vasculares.

\section{Consideraciones éticas}

Se clasificó el estudio como una investigación de riesgo leve y se obtuvo la autorización de acuerdo con los protocolos de Helsinki y la Ley 23 de I98I sobre el código de ética médica. El procedimiento establecido en este estudio solo se utilizó para el mismo y, en ningún momento, se compartió o empleó en otros proyectos. Se garantizó la confidencialidad de la información, y prevaleció el criterio del respeto de la dignidad, y la protección de los derechos y el bienestar de los pacientes.

Consentimiento informado: Se obtuvo consentimiento informado para todos los procedimientos, después de explicar a los pacientes o sus cuidadores las ventajas y riesgos de la intervención.

Conflictos de interés: En el presente estudio los autores declaran no tener ningún conflicto de interés.

Fuente de financiación: Propia de los autores.

\section{Referencias}

I. Kern S, Niemeyer C, Darge K, Merz C, Laudenberger J, Uhl M. Differentiation of vascular birthmarks by MR imaging. An investigation of hemangiomas, venous and lymphatic malformations. Acta Radiol. 2000;4I:453-7.
2. Redondo P. Malformaciones vasculares (I). Concepto, clasificación, fisio patogenia y manifestaciones clínicas Actas Dermosifiliogr. 2007;98:I4I-58.

3. Jimenez CE, Randial L, Rozo JP, Vélez N. Malformación vascular hepática con falla cardiaca secundaria en un infante de dos meses, manejada por vía endovascular con onyx, presentación de un caso y revisión de la literatura. Pediatr. 2015;48:I06-I2.

https://doi.org/IO.IOI6/j.rcpe.20I5.I2.002

4. Finn MC, Glowacki J, Mulliken JB. Congenital vascular lesions: clinical application of a new classification. J Pediat Surg. 1983;18:894-900.

https://doi.org/IO.IOI6/s0022-3468(83)80043-8

5. Belov S. Classification of congenital vascular defects. Int Angiol. 1990;9:I4I-6.

6. Lidsky ME, Markovic JN, Miller MJ Jr, Shortell CK. Analysis of the treatment of congenital vascular malformations using a multidisciplinary approach. J Vasc Surg. 2012;56:1355-62.

https://doi.org/IO.IOI6/j.jvs.20I2.04.066

7. Rokni-Yazdi H, Ghajarzadeh M, Keyvan AH, Namavar MJ, Azizi S. Peripheral venous malformations with a dominant outflow vein: results of ethanol embolization. Acta Medica Iranica. 20I4;52:868-7I.

8. Yun W, Kim Y, Lee K, Kim D. Predictors of response to percutaneous etanol sclerotherapy (PES) in patients with venous malformations: Analysis of patient self-assessment and imaging. J Vasc Surg. 2009;50:58I-9.

9. Lidsky M, Markovic J, Miller M, Shortell C. Analysis of the treatment of congenital vascular malformations using a multidisciplinary approach. J Vasc Surg. 2012;56:I355-62. https://doi.org/IO.IOI6/j.jvs.2012.04.066

Io. Lee BB, Do YS, Byun HS, Choo IW, Kim DI, Huh SH. Advanced management of venous malformation with ethanol sclerotherapy: Mid-term results. J Vasc Surg. 2003;37:533-8. https://doi.org/I0.I067/mva.2003.9I

II. Beverly CK, San CY, Lau EY, Yu SC, Burd A. Multidisciplinary vascular malformations clinic in Hong Kong. Hong Kong Med J. 2013;19:II6-23.

I2. Park HS, Do YS, Kwang BP, Kim DI, Kim YD, Yim MJ, et al. Ethanol embolotherapy of hand arteriovenous malformations. J Vasc Surg. 20II;53:725-3I. https://doi.org/IO.IOI6/j.jvs.20I0.09.028

13. Cho SK, Do YS, Kim DI, Kim YW, Shin SW, Park KB, et al. Peripheral arteriovenous malformations with a dominant outflow vein: Results of ethanol embolization. Korean J Radiol. 2008;9:258-67. https://doi.org/I0.3348/kjr.2008.9.3.258

I4. Hyun D, Do YS, Park KB, Kim DI, Kim YW, Park HS, et al. Ethanol embolotherapy of foot arteriovenous malformations. J Vasc Surg. 2013;58:1619-26. https://doi.org/IO.IoI6/j.jvs.2013.06.074 
I5. Sharma D, Lamba S, Pandita A, Shastri S. KlippelTrénaunay Syndrome - A very rare and interesting syndrome. Clin Med Insights Circ Respir Pulm Med. 2015;9:I-4. https://doi.org/Io.4I37/CCRPM.S2I645

I6. Klippel M, Trénaunay P. Du noevus variquex osteohypertrophiques. Arch of Gen Med. 1900;3:64I-72.

I7. Nitecki S, Bass A. Ultrasound-guided foam sclerotherapy in patients with Klippel-Trenaunay syndrome. Isr Med Assoc J. 2007;9:72-5.

I8. Noel AA, Gloviczki P, Cherry KJ, Rooke TW, Stanson AW, Driscoll DJ. Surgical treatment of venous malformations in Klippel-Trénaunay síndrome. J Vasc Surg. 2000;32:840-7. https://doi.org/IO.IO67/mva.2000.II0343

I9. Wang ZK, Wang FY, Zhu RM, Liu J. KlippelTrenaunay syndrome with gastrointestinal bleeding, splenic hemangiomas and left inferior vena cava. World J Gastroenterol. 20I0;16:1548-52. https://doi.org/IO.3748/wjg.vi6.ir2.1548

20. Gloviczki P, Stanson AW, Stickler GB, Johnson CM, Toomey BJ, Meland NB, et al. Klippel-Trenaunay syndrome: the risks and benefits of vascular interventions. Surgery. I991;IIO:469-79.

2I. Deshpande DH, Vidyasagar C. Histology of the persistent embryonic veins in arteriovenous malformations of brain. Acta Neurochir (Wien). 1980;53:227-36.

22. Eifert S, Villavicencio JL, Kao TC, Taute BM, Rich NM. Prevalence of deep venous anomalies in congenital vascular malformations of venous predominance. J Vasc Surg. 2000;3I:462-7I.

23. Krämer D, Muñoz P, Alfaro C, Araníbar L, Cárdenas L, Morovic CG, et al. Propanolol en el tratamiento de los hemangiomas de la infancia. Rev Chil Pediatr. 2010;8I:523-30.

http://dx.doi.org/I0.4067/So370-41062010000600006

24. Enjolras O. Classification and management of the various superficial vascular anomalies: Hemangiomas and vascular malformations. J Dermatol. 1997;24:70I-IO. https://doi.org/IO.IIII/j.I346-8I38.1997.tbo2522.x

25. Frieden IJ, Haggstrom AN, Drolet BA, Mancini AJ, Friedlander SF, Boon L, et al. Infantile hemangiomas: current knowledge, future directions. Proceedings of a research workshop on infantile hemangiomas. Pediatr Dermatol. 2005;22:383-406. https://doi.org/IO.IIII/j.I525-I470.2005.00I02.x

26. MacFie CC, Jeffery SL. Diagnosis of vascular skin lesions in children: An audit and review. Pediatr Dermatol. 2008;25:7-I2. https://doi.org/I0.IIII/j.I525-I470.2007.00573.x

27. Legiehn GM, Heran MK. Classification, diagnosis, and interventional radiologic management of vascular malformations. Orthop Clin North Am. 2006;37:435-74. https://doi.org/Io.IoI6/j.ocl.2006.04.005
28. Lee BB, Baumgartner I, Berlien P, Bianchini G, Burrows $\mathrm{P}$, Gloviczki P, et al. Diagnosis and treatment of venous malformations. Consensus document of the International Union of Phlebology (IUP): updated 20I3. Int Angiol. 20I5;34:97-I49.

29. Puig S, Aref H, Chigot V, Bonin B, Brunelle F. Classification of venous malformations in children and implications for sclerotherapy. Pediatr Radiol. 2003;33:99-I03. https://doi.org/IO.IO07/s00247-002-0838-9

30. Lee BB, Laredo J, Kim YW, Neville R. Congenital vascular malformations: general treatment principles. Phlebology. 2007;22:258-63. https://doi.org/IO.I258/026835507782655I82

3I. Yakes WF, Luethke J, Parker SH, Stavros AT, Rak KM, Hopper KD, et al. Etanol embolization of vascular malformations. Radiographics. 1990;10:787-96. https://doi.org/IO.II48/radiographics.IO.5.22I797I

32. Loose DA. Surgical management of venous malformations. Phlebology 2007;22:276-82. https://doi.org/IO.I258/026835507782655254

33. Baek HJ, Hong JP, Choi JW, Suh DC. Direct percutaneous alcohol sclerotherapy for venous malformations of head and neck region without fluoroscopic guidance: Technical consideration and outcome. Neurointervention. 20II;6:84-8. https://doi.org/IO.5469/neuroint.20II.6.2.84

34. Bae S, Do YS, Shin SK, Park KB, Kim DI, Kim YW, et al. Ethanol embolotherapy of pelvic arteriovenous malformations: an initial experience. Korean J Radiol. 2008;9:I48-I54. https://doi.org/IO.3348/kjr.2008.9.2.I48

35. Su L, Wang D, Han Y, Wang Z, Zheng L, Fan X. Absolute ethanol embolization of infiltrating-diffuse extracranial arteriovenous malformations in the head and neck. Eur J Vasc Endovasc Surg. 2015;50:II4e-2I. https:// doi.org/IO.IOI6/j.ejvs.2015.02.020

36. Zakhari S. Overview: How is alcohol metabolized by the body? Alcohol Res Health. 2006;29:245-54.

37. Zheng LZ, Fan XD, Zheng JW, Su LX. Ethanol embolization of auricular arteriovenous malformations: Preliminary results of 17 cases. AJNR Am J Neuroradiol. 2009;30:I679-84. https://doi.org/Io.3174/ajnr.Aı687

38. Cai MJ, Liang WX, Mai WW, He WL, Kuang HX. Clinical effects and safety of interventional therapy for parenchyma vascular malformation. Gen Mol Res. 20I4;I3:I735-43. https://doi.org/I0.4238/20I4.January.22.13

39. Lee B, Kim D, Huh M, Kim H, Choo W, Byun HS. New experiences with absolute ethanol sclerotherapy in the management of a complex form of congenital venous malformation. J Vasc Surg. 200I;33:764-72. https://doi.org/IO.IO67/mva.200I.II2209 
40. Tan KT, Kirby J, Rajan DK, Hayeems E, Beecroft JR, Simons ME. Percutaneous sodium tetradecyl sulfate sclerotherapy for peripheral venous vascular malformations: A single-center experience. J Vasc Interv Radiol. 2007;I8:343-5I.

https://doi.org/Io.IoI6/j.jvir.2006.I2.735

4I. Zanjani K, Mazloumi M, Zeinaloo A, Hedayati M, Khalilzadeh O, Yazdi H. Transcatheter embolization of congenital hepatic arteriovenous malformation using ethylene-vinyl alcohol copolymer (Onyx). Diagn Interv Radiol. 2012;18:23I-35.

https://doi.org/I0.426I/1305-3825.DIR.44I8-II.O

42. Bolus C, Yamada R, Alshora S, Hannegan C. Transarterial embolization of a hepatic arteriovenous mal- formation in an infant using Onyx: A case report and review of the differential diagnosis imaging findings. J Radiol Case Rep. 20I4;8:33-42.

https://doi.org/I0.394I/jrcr.v8i8.2I7I

43. Szajner M, Roman T, Markowicz J, Szczerbo-Trojanowski M. Onyx ${ }^{\circledR}$ in endovascular treatment of cerebral arteriovenous malformations - a review. Pol J Radiol. 2013;78:35-4I. https://doi.org/IO.I2659/PJR.889120

44. Orlando J, Mendes J, Galva H, Campos A, Nishinari $\mathrm{K}$, Wolosker N. Outpatient percutaneous treatment of deep venous malformations using pure ethanol at low doses under local anesthesia. Clinics. 2010;65:837-40. https://doi.org/I0.I590/SI807-593220I0000900004 